Robert C. Dalang, Département de Mathématiques, Ecole Polytechnique

Fédérale, 1015 Lausanne, Switzerland, email:dalang@@math.epfl.ch

T. Mountford $\dagger$ Department of Mathematics, University of California, Los

Angeles, CA 90024, U.S.A., email: malloy@@math.ucla.edu

\title{
POINTS OF INCREASE OF FUNCTIONS IN THE PLANE
}

\begin{abstract}
The set of continuous functions of a single nonnegative real variable with at least one point of increase is a "small" set under two natural definitions: on the one hand, it is a set of first Baire category, and on the other hand, according to a famous result of Dvoretsky, Erdös and Kakutani, it is also a set with Wiener measure zero. In this paper, an analogous question for continuous functions of two nonnegative real variables is examined. Consider the set of continuous functions $f$ on the nonnegative quadrant for which there exists a monotone curve along which the restriction of $f$ has a point of increase. In this paper, it is shown that this set is "small" in the sense that it is of first Baire category. However, this set is "large" in the sense that it has full measure under the probability measure induced by the standard Brownian sheet.
\end{abstract}

\section{Introduction}

A real-valued function $u \mapsto \alpha(u)$ defined on an interval of the real line $\mathbb{R}$ is said to have a point of increase at $u_{0}$ if there is $\delta>0$ such that $\alpha(u)<\alpha\left(u_{0}\right)$ for $u \in\left[u_{0}-\delta, u_{0}\left[\right.\right.$ and $\alpha(u)>\alpha\left(u_{0}\right)$ for $\left.\left.u \in\right] u_{0}, u_{0}+\delta\right]$; we will say that $u_{0}$ is a wide sense point of increase of $\alpha(\cdot)$ if there is $\delta>0$ such that $\alpha(u) \leq \alpha\left(u_{0}\right)$ for $u \in\left[u_{0}-\delta, u_{0}\right]$ and $\alpha(u) \geq \alpha\left(u_{0}\right)$ for $u \in\left[u_{0}, u_{0}+\delta\right]$. Points of decrease and wide sense points of decrease are defined analogously.

\footnotetext{
Key Words: Points of increase, Baire Category, Brownian sheet, Brownian motion

Mathematical Reviews subject classification: Primary: 26B35 Secondary: 28C20, 60G60

Received by the editors November 20, 1996

* This research was was partially supported by ARO grant DAAH-04-94-G-0261.

$\dagger$ The research of this author is partially supported by NSF grant DMS-9157461 and by the Sloan Foundation.
} 
Though any smooth (non-constant) function obviously has many points of increase or decrease, it is well-known that the set $\mathcal{I}_{1}$ of real-valued functions defined on $\mathbb{R}_{+}$that admit a point of increase or decrease is a small set. Indeed, a famous result of Dvoretsky, Erdös and Kakutani [5] asserts that the Wiener measure of $\mathcal{I}_{1}$ is zero, or equivalently, with probability one, sample paths of a Brownian motion have no points of increase or decrease.

A natural question is whether or not the set $\mathcal{I}_{1}$ is small in the topological sense of Baire category. Bruckner and Garg [3] extended a result of Jarnik [6] and showed that $\mathcal{I}_{1}$ is of first Baire category, that is, typical functions have no points of increase or decrease. Recent accounts of these proofs are contained in the books of Bruckner [1, Chapter 13] and Bruckner, Bruckner and Thomson [2, Section 10.6].

In this paper, motivated by the results of [4] concerning the random function of two variables known as the Brownian sheet, we examine the questions analogous to those above for functions of two non-negative real variables. No knowledge of properties of the Brownian sheet are needed in this paper, but for completeness, we recall that the Brownian sheet is one of the natural extensions of Brownian motion to functions of two real variables, more precisely, it is the mean-zero continuous Gaussian process $W=\left(W(t), t \in \mathbb{R}_{+}^{2}\right)$, with the covariance

$$
\operatorname{Cov}(W(s) W(t))=\min \left(s_{1}, t_{1}\right) \min \left(s_{2}, t_{2}\right)
$$

for all $s=\left(s_{1}, s_{2}\right)$ and $t=\left(t_{1}, t_{2}\right)$ in $\mathbb{R}_{+}^{2}$. This stochastic process induces a probability measure on the set $C\left(\mathbb{R}_{+}^{2}, \mathbb{R}\right)$ of continuous functions on $\mathbb{R}_{+}^{2}$, that we call Brownian sheet measure.

Consider now the set $\mathcal{I}_{2}$ of real-valued functions $f$ defined on $\mathbb{R}_{+}^{2}$ for which there exists a monotone path along which $f$ has a point of increase (precise definitions are given in Section 2). Again, one can ask whether this set is large or small. In [4], it was proved that sample paths of the Brownian sheet belong to $\mathcal{I}_{2}$ almost surely, or equivalently, the set $\mathcal{I}_{2}$ has measure 1 under Brownian sheet measure. In this probabilistic sense, the set $\mathcal{I}_{2}$ is large.

The objective of this paper is to determine whether or not $\mathcal{I}_{2}$ is also large in the topological sense of Baire category. We shall prove that in fact, the set $\mathcal{I}_{2}$ has first Baire category, and therefore $\mathcal{I}_{2}$ is small in the topological sense. This proves in particular that for a typical function in $C\left(\mathbb{R}_{+}^{2}, \mathbb{R}\right)$, there is no monotone path along which the function has a point of increase. Even though these functions are the rule rather than the exception, we are not aware of any explicit construction of a function with these properties.

We note that the results of this paper remain valid (along with the proofs) if $\mathbb{R}_{+}^{2}$ is replaced by $\mathbb{R}^{2}$. 


\section{Terminology}

The set $\mathbb{R}_{+}^{2}$ is endowed with the (partial) order $\leq$ defined by

$$
s=\left(s_{1}, s_{2}\right) \leq t=\left(t_{1}, t_{2}\right) \quad \Longleftrightarrow \quad s_{1} \leq t_{1} \text { and } s_{2} \leq t_{2} .
$$

A convenient norm on $\mathbb{R}^{2}$ is $|t|=\left|t_{1}\right|+\left|t_{2}\right|$. An increasing curve is a totally ordered and connected subset of $\mathbb{R}_{+}^{2}$. A (canonically parameterized) increasing path (resp. decreasing path) $\gamma$ is a continuous function defined on some interval of $\mathbb{R}$ with values in $\mathbb{R}_{+}^{2}$ with the property that $\gamma(u) \leq \gamma(v)($ resp. $\gamma(u) \geq \gamma(v)$ ) when $u \leq v$ and $|\gamma(u)-\gamma(v)|=|u-v|$. This last condition implies that the map $\gamma$ is one-to-one. Recall [8, Theorem 2.7] that a set is an increasing curve if and only if it is the image of an increasing path. Moreover, increasing or decreasing paths are Lipschitz functions, therefore, when equipped with the topology of uniform convergence, the set of increasing or decreasing paths defined on a compact interval with values in a compact set is compact.

It is also natural to consider another (partial) order $\unlhd$ on $\mathbb{R}_{+}^{2}$ defined by

$$
s=\left(s_{1}, s_{2}\right) \unlhd t=\left(t_{1}, t_{2}\right) \quad \Longleftrightarrow \quad s_{1} \leq t_{1} \text { and } s_{2} \geq t_{2} .
$$

The definitions of increasing curve and increasing or decreasing path relative to the (partial) order $\unlhd$ also make sense and the properties mentioned above remain valid. A curve that is increasing or a path that is increasing or decreasing with respect to either of the (partial) orders $\leq$ or $\unlhd$ is termed a monotone curve or a monotone path.

A function $f \in C\left(\mathbb{R}_{+}^{2}, \mathbb{R}\right.$ ) has a point of increase (resp. a wide sense point of increase) along a monotone path $\gamma$ if the function $f \circ \gamma$ admits a point of increase (resp. a wide sense point of increase).

\section{The Baire category argument}

Consider the space $C\left(\mathbb{R}_{+}^{2}, \mathbb{R}\right)$ of continuous real-valued functions on $\mathbb{R}_{+}^{2}$, equipped with the topology of uniform convergence on compact sets. This space is a complete metric space, so by the Baire Category Theorem [7, Chapter 7.7], it is not a set of first Baire category.

Let $\mathcal{I}_{2}$ be the set of functions $f \in C\left(\mathbb{R}_{+}^{2}, \mathbb{R}\right)$ with the following property:

there exists $r>0$ and a monotone path $\gamma:[-r, r] \rightarrow \mathbb{R}_{+}^{2}$ such that $f(\gamma(-x)) \leq f(\gamma(0)) \leq f(\gamma(x))$, for all $x \in[0, r]$.

The main result of this paper is the following. 
Theorem 1 The set $\mathcal{I}_{2}$ is of first Baire category, that is, for a typical function $f$ in $C\left(\mathbb{R}_{+}^{2}, \mathbb{R}\right)$, there is no monotone path along which $f$ has a wide sense point of increase.

In order to show that $\mathcal{I}_{2}$ is a set of first Baire category, we must show that $\mathcal{I}_{2}$ is a countable union of closed nowhere dense sets (recall that a subset of $C\left(\mathbb{R}_{+}^{2}, \mathbb{R}\right)$ is nowhere dense if its complement is dense in $C\left(\mathbb{R}_{+}^{2}, \mathbb{R}\right)$ ). Note that

$$
\mathcal{I}_{2}=\bigcup_{r>0, n \in \mathbb{N}} I(r, n)
$$

where

$$
\begin{aligned}
I(r, n)=\{f \in & C\left(\mathbb{R}_{+}^{2}, \mathbb{R}\right): \text { there exists a monotone path } \gamma:[-r, r] \rightarrow \mathbb{R}_{+}^{2} \\
& \text { such that }|\gamma(0)| \leq n \text { and } f(\gamma(-x)) \leq f(\gamma(0)) \leq f(\gamma(x)), \\
& \text { for all } x \in[0, r]\} .
\end{aligned}
$$

We recall that $|\gamma( \pm r)-\gamma(0)|=r$, so the monotone path in the definition of $I(r, n)$ has length $r$ at least.

Lemma 2 For $n \in \mathbb{N}$ and $r>0$, the set $I(r, n)$ is closed in $C\left(\mathbb{R}_{+}^{2}, \mathbb{R}\right)$.

Proof. Fix $n \in \mathbb{N}$ and $r>0$. Let $f \in C\left(\mathbb{R}_{+}^{2}, \mathbb{R}\right)$ and $\left(f_{i}, i \in \mathbb{N}\right)$ be a sequence of elements of $I(r, n)$ such that $f_{i}$ converges uniformly on compact sets to $f$. We must show that $f \in I(r, n)$.

Because $f_{i} \in I(r, n)$, there is a monotone path $\gamma_{i}:[-r, r] \rightarrow \mathbb{R}_{+}^{2}$ such that $\left|\gamma_{i}(0)\right| \leq n$ and

$$
f_{i}\left(\gamma_{i}(-x)\right) \leq f_{i}\left(\gamma_{i}(0)\right) \leq f_{i}\left(\gamma_{i}(x)\right), \quad \text { for all } x \in[0, r] .
$$

By the compactness property of the set of monotone paths mentioned in Section 2 , there is a subsequence $\left(\gamma_{i_{j}}\right)$ that converges uniformly on $[-r, r]$ to a monotone path $\gamma$. Because $f_{i_{j}}$ converges uniformly to $f$ on $[0, n+r]^{2}$, we can pass to the limit in (1) to get $|\gamma(0)| \leq n$ and

$$
f(\gamma(-x)) \leq f(\gamma(0)) \leq f(\gamma(x)), \quad \text { for all } x \in[0, r] .
$$

Consequently, $f$ belongs to $I(r, n)$.

Lemma 3 For $n \in \mathbb{N}$ and $r>0$, the complement of $I(r, n)$ is dense in $C\left(\mathbb{R}_{+}^{2}, \mathbb{R}\right)$. 
Proof. Because the set $\mathcal{L}\left(\mathbb{R}_{+}^{2}, \mathbb{R}\right)$ of Lipschitz continuous functions on $\mathbb{R}_{+}^{2}$ is dense in $C\left(\mathbb{R}_{+}^{2}, \mathbb{R}\right)$, it suffices to show that for all $f \in \mathcal{L}\left(\mathbb{R}_{+}^{2}, \mathbb{R}\right)$, for all $n \in \mathbb{N}$ and for all $\eta>0$, there exists $g \in C\left(\mathbb{R}_{+}^{2}, \mathbb{R}\right) \backslash I(r, n)$ such that $|f(t)-g(t)|<\eta$ for all $t \in[0, n]^{2}$. For given $f, n$ and $\eta$, we shall take $g$ to be of the form

$$
g(t)=f(t)+\eta h\left(\frac{t}{\delta}\right)
$$

where the function $h$ is defined below and $\delta$ is chosen appropriately. The function $h$ will be such that $g \notin I(r, n)$ and $|h| \leq 1$. This will complete the proof of Lemma 3.

\section{The construction of the function $h$}

For $t \in \mathbb{N}^{2}$ and $\varepsilon>0$, let $\partial B(t, \varepsilon)$ denote the circle of radius $\varepsilon$ centered at $t$. Set

$$
C^{e}(k, l)=\partial B\left((2 k, 2 l), \frac{\sqrt{2}}{2}\right), \quad C^{o}(k, l)=\partial B\left((2 k+1,2 l+1), \frac{\sqrt{2}}{2}\right) .
$$

Note that $C^{e}(k, l)$ has a single point of intersection with the four circles $C^{o}(k-$ $1, l-1), C^{o}(k-1, l), C^{o}(k, l-1)$, and $C^{o}(k, l)$, with a $\pm 45^{\circ}$ tangent at this point of intersection, and $C^{e}(k, l)$ is disjoint from all other $C^{o}\left(k^{\prime}, l^{\prime}\right)$, and from $C^{e}\left(k^{\prime}, l^{\prime}\right)$ if $\left(k^{\prime}, l^{\prime}\right) \neq(k, l)$. Set

$$
C=\bigcup_{(k, l) \in \mathbb{N}^{2}}\left(C^{e}(k, l) \cup C^{o}(k, l)\right) .
$$

A portion of the set $C$ is shown in Figure 1 .

Let $t_{k, l}=\left(k+\frac{1}{2}, l+\frac{1}{2}\right)$. Observe that

$$
D=\left\{t_{k, l},(k, l) \in \mathbb{N}^{2}\right\}
$$

is the set of points that belong to more than one of the circles $C^{e}\left(k^{\prime}, l^{\prime}\right)$ or $C^{o}\left(k^{\prime}, l^{\prime}\right)$.

For $s$ and $t$ in $\mathbb{R}^{2}$, let $d(s, t)$ denote the Euclidean distance between $s$ and $t$. For $F \subset \mathbb{R}^{2}$ and $t \in \mathbb{R}^{2}$, let

$$
d(t, F)=\inf \{d(t, s), s \in F\},
$$

and for $\varepsilon>0$, we set

$$
F_{\varepsilon}=\left\{t \in \mathbb{R}^{2}: d(t, F)<\varepsilon\right\} .
$$

The diameter of $F$ is the number $\sup _{s, t \in F} d(s, t)$. 


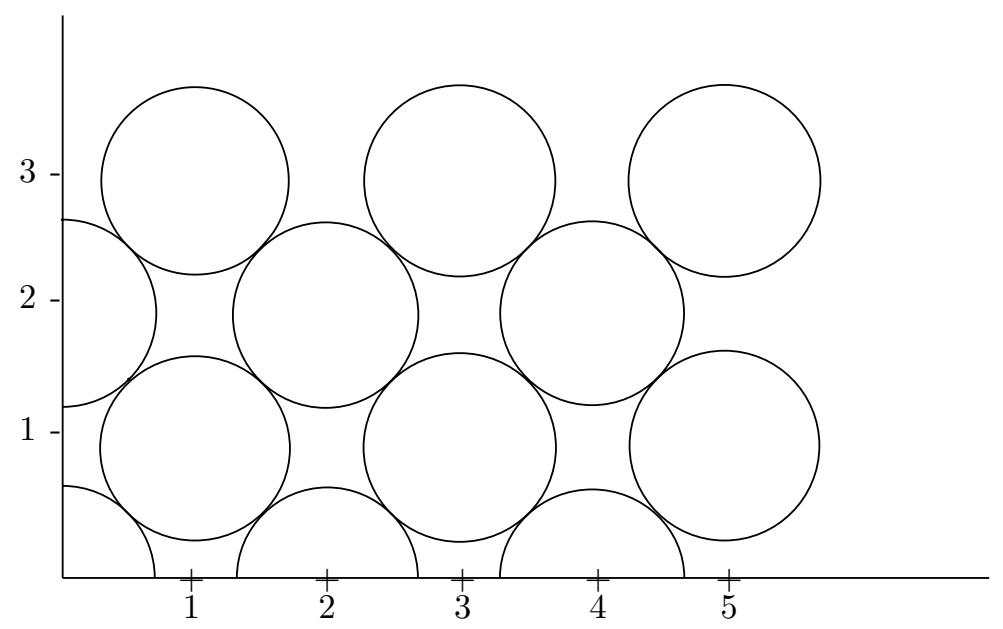

Figure 1: The set $C$.

Lemma 4 (a) There is $\left.\varepsilon_{0} \in\right] 0,1\left[\right.$ such that for all distinct $t, t^{\prime} \in D$, there is no monotone curve contained in $C_{\varepsilon_{0}}$ that has one extremity within $\varepsilon_{0}$ of $t$ and the other within $\varepsilon_{0}$ of $t^{\prime}$.

(b) For $0<\varepsilon<\frac{1}{4} \varepsilon_{0}^{2}, C_{\varepsilon}$ contains no monotone curve with length $\geq 3$.

Proof. Observe that $C \backslash D$ is a countable union of connected components, each of which is an quarter circle (without the two extremities) whose diameter is $\leq 1$. It therefore suffices to prove (a) for fixed $t, t^{\prime} \in D$ such that $\left|t-t^{\prime}\right|=1$.

It is clear from the definition of $C$ that if $t, t^{\prime} \in D$ are such that $\left|t-t^{\prime}\right|=1$, then there is no monotone curve contained in $C$ with extremities $t$ and $t^{\prime}$. Suppose by contradiction that there were no $\varepsilon_{0}>0$ with the property stated in (a). Then for all $\varepsilon>0$, there would be a monotone path $\gamma_{\varepsilon}$ with one extremity within $\varepsilon$ of $t$ and the other within $\varepsilon$ of $t^{\prime}$, and with range contained in $C_{\varepsilon}$. By the compactness property of monotone paths mentioned in Section 2 , there would be a sequence $\varepsilon_{n} \downarrow 0$ such that $\gamma_{\varepsilon_{n}}$ would converge uniformly to a monotone path $\gamma$. This monotone path would have extremities $t$ and $t^{\prime}$ and its range would be contained in $C$, a contradiction. This proves (a).

Before proving (b), note that elementary geometric considerations yield the following: for any $\varepsilon>0, C \backslash D_{\sqrt{4 \varepsilon}}$ is a union of closed components, each with diameter $\leq 1$, and the distance between two such components is at least $2 \varepsilon$. Therefore, $C_{\varepsilon} \backslash D_{\sqrt{4 \varepsilon}}$ is also a union of disjoint components, each with 
diameter $\leq 1$

To prove (b), fix $0<\varepsilon<\frac{1}{4} \varepsilon_{0}^{2}$ and suppose that $\gamma$ is a monotone curve of length $\geq 3$ contained in $C_{\varepsilon}$. Because the components of $C_{\varepsilon} \backslash D_{\sqrt{4 \varepsilon}}$ have diameter $\leq 1, \gamma$ must meet at least three distinct components of this set, and therefore, there are two distinct points $t, t^{\prime} \in D$ such that $\gamma$ passes within $\sqrt{4 \varepsilon}$ of $t$ and $t^{\prime}$. Because $\sqrt{4 \varepsilon}<\varepsilon_{0}$ and $\varepsilon<\frac{1}{4} \varepsilon_{0}^{2}<\varepsilon_{0}$, this contradicts (a).

Define a function $h: \mathbb{R}_{+}^{2} \rightarrow \mathbb{R}_{+}$by the formula

$$
h(t)=\max \left(0,1-\frac{d(t, C)}{\varepsilon_{0}}\right) .
$$

Clearly, $h$ is continuous, takes the value 1 on $C$ and 0 on $\mathbb{R}_{+}^{2} \backslash C_{\varepsilon_{0}}$.

Proposition 5 For any monotone path $\gamma:[-6,6] \rightarrow \mathbb{R}_{+}^{2}$, either

$$
\sup _{x \in[-6,0]} h(\gamma(x))-h(\gamma(0)) \geq \frac{1}{2} \text { or } \inf _{x \in[0,6]} h(\gamma(x))-h(\gamma(0)) \leq-\frac{1}{2} .
$$

Proof. Note that all connected components of $\mathbb{R}_{+}^{2} \backslash C$ have diameter $\leq 2$. Let $t=\gamma(0)$ and suppose first that $d(t, C) \geq \varepsilon_{0} / 2$. Then $h(t) \leq \frac{1}{2}$. Because $|\gamma(-6)-\gamma(0)|=6, d(\gamma(-6), \gamma(0)) \geq 3$, so there is $x \in[-6,0]$ such that $\gamma(x) \in C$, and therefore

$$
h(\gamma(x))-h(\gamma(0))=1-h(\gamma(0)) \geq \frac{1}{2},
$$

that is, the first inequality in (2) holds.

Now suppose that $d(t, C)<\varepsilon_{0} / 2$. Then $h(t)>\frac{1}{2}$. Because $|\gamma(6)-\gamma(0)|=$ $6, d(\gamma(6), \gamma(0)) \geq 3$, so Lemma 4 (b) implies that there is $x \in[0,6]$ such that $\gamma(x) \notin C_{\varepsilon_{0}}$, that is,

$$
h(\gamma(x))-h(\gamma(0))=0-h(\gamma(0)) \leq-\frac{1}{2},
$$

and so the second inequality in (2) holds.

\section{End of the proof of Lemma 3}

Fix $f \in \mathcal{L}\left(\mathbb{R}_{+}^{2}, \mathbb{R}\right), n \in \mathbb{N}, r>0$ and $\eta>0$. Let $M>0$ be such that

$$
|f(t)-f(s)| \leq M|t-s| \quad \text { if } s, t \in[0, n+r]^{2} .
$$


Fix $\delta>0$ such that

$$
\delta \leq \min \left(\frac{r}{6}, \frac{\eta}{18 M}\right)
$$

and set

$$
g(t)=f(t)+\eta h\left(\frac{t}{\delta}\right) .
$$

Let $\gamma:[-r, r] \rightarrow \mathbb{R}_{+}^{2}$ be any monotone path such that $|\gamma(0)| \leq n$. Note that if $|x|<6 \delta$, then $|x|<r$ and

$$
|f(\gamma(x))-f(\gamma(0))| \leq M|\gamma(x)-\gamma(0)| \leq M \cdot 6 \delta \leq \frac{\eta}{3} .
$$

On the other hand, the range of the function $x \mapsto \gamma(x) / \delta$ is a monotone curve and $|\gamma( \pm 6 \delta) / \delta-\gamma(0) / \delta|=6$. By Proposition 5,

$$
\sup _{x \in[-6 \delta, 0]} \eta\left(h\left(\frac{\gamma(x)}{\delta}\right)-h\left(\frac{\gamma(0)}{\delta}\right)\right) \geq \frac{\eta}{2}
$$

or

$$
\inf _{x \in[0,6 \delta]} \eta\left(h\left(\frac{\gamma(x)}{\delta}\right)-h\left(\frac{\gamma(0)}{\delta}\right)\right) \leq-\frac{\eta}{2}
$$

Therefore, either

$$
\sup _{x \in[-6 \delta, 0]} g(\gamma(x))-g(\gamma(0)) \geq-\eta / 3+\eta / 2>0
$$

or

$$
\inf _{x \in[0,6 \delta]} g(\gamma(x))-g(\gamma(0)) \leq \eta / 3-\eta / 2<0 .
$$

This implies that $g \notin I(r, n)$ and, and because $|f(t)-g(t)|<\eta$ for all $t \in[0, n]^{2}$, the proof of Lemma 3 is complete.

\section{Proof of Theorem 1}

As already observed, the set $C\left(\mathbb{R}_{+}^{2}, \mathbb{R}\right)$ is not a set of first Baire category. On the other hand, the set $\mathcal{I}_{2}$ is the union of the sets $I(r, n)$, and this union can be made countable by only considering rational values of $r>0$. By Lemmas 2 and 3 , each of these sets is closed and nowhere dense, therefore $\mathcal{I}_{2}$ is a set of first Baire category. This proves Theorem 1.

ACKnowledgement. The authors thank John B. Walsh who, upon learning the result of [4], first raised the question of whether or not all continuous functions of two variables admit points of increase along monotone curves. References [1], [2] and [3] were supplied by Brian S. Thomson. 


\section{References}

[1] A. M. Bruckner, Differentiation of Real Functions, Springer-Verlag, Lecture Notes in Math. 659, (1978).

[2] A. M. Bruckner, J. B. Bruckner and B. S. Thomson, Real Analysis, (1996) Prentice Hall.

[3] A. M. Bruckner, and K. Garg, The level set structure of a residual set of continuous functions, Trans. Amer. Math. Soc. (1977) 232, 307-321.

[4] R. C. Dalang and T. Mountford, Points of increase of the Brownian sheet, Prob. Theory and Related Fields (1997) to appear.

[5] A. Dvoretsky, P. Erdös and S. Kakutani,Non-increase everywhere of the Brownian motion process Proc. Fourth Berkeley Symp. Math. Stat. Prob. (1961) 2, 103-116.

[6] V. Jarkik, Uber die differenzierbarkeit stetiger funktionen, Fund. Math. (1933) 21, 48-58.

[7] H. L. Royden, Real Analysis, Second Edition (1968) Macmillan, London.

[8] J. B. Walsh, Optional increasing paths, Processus Aléatoires à Deux Indices (H. Korezlioglu, G. Mazziotto and J. Szpirglas, eds), SpringerVerlag, Lecture Notes in Math. (1981) 863, 172-201. 